\title{
Análisis Microbiológico de Biosólidos para su aprovechamiento en cultivos de tallo corto
}

\section{Microbiological Analysis of Biosolids for use in short stem crops}

\author{
SOTO-ALCOCER, José Luis†๋*, RIVERA-MOSQUEDA, Ma. Cruz, GONZÁLEZ-PONCE, María del \\ Refugio y AYALA-ISLAS, Alberto
}

Instituto Tecnológico Superior de Irapuato, Carr. Irapuato-Silao, El Copal, 36821 Irapuato, Gto

ID $1^{\text {er }}$ Autor: José Luis, Soto-Alcocer / ORC ID: 0000-0003-3435-9057, Researcher ID Thomson: S-4783-2018, CVU

CONACYT ID: 80822

ID $1^{\text {er }}$ Coautor: Ma. Cruz, Rivera-Mosqueda / ORC ID: 0000-0001-7752-9226, Researcher ID Thomson: Y-2422-2019, CVU CONACYT ID: 333557

ID $2^{\text {do }}$ Coautor: María del Refugio, González-Ponce / ORC ID: 0002-1170-2126, Researcher ID Thomson: S-4503-2018, CVU CONACYT ID: 327208

ID $3^{\text {er }}$ Coautor: Alberto, Ayala-Islas / ORC ID: 0000-0001-8613-9297, Researcher ID Thomson: Y-2360-2019, CVU CONACYT ID: 162442

DOI: $10.35429 / J B E B .2019 .7 .3 .31 .38$

Recibido 10 de Enero, 2019; Aceptado 30 Marzo, 2019

\begin{abstract}
Resumen
Se analizaron microbiológicamente muestras de lodos y biosólidos de acuerdo a la NOM-004-SEMARNAT-2002, del Sistema Intermunicipal de Tratamiento y Disposición de Aguas Residuales de los municipios del Rincón (SITRATA) durante un lapso de 10 meses en el año 2017, la mayoría se encontró dentro de los L.M.P. según la normatividad. Además, se detectó que el uso de ácido peracético en dosis mayores que $0.1 \%$ v/v puede resultar una alternativa de desinfección en su uso en lodos, para disminuir la carga microbiológica, antes de ser utilizados en cultivos. Se realizaron análisis fisicoquímicos a muestras de lodo y suelo para determinar su contenido de $\mathrm{N}$ y P, encontrándose que el lodo sólido cuenta con 1.19 $\%$ de $\mathrm{N}$ y $3.51 \%$ de $\mathrm{P}_{2} \mathrm{O}_{5}$ determinado en base seca; que corresponden a valores altos de estos macronutrientes, indispensables para los cultivos. Se realizaron pruebas en invernadero con dosis de aplicación de 0 a 100 en porcentaje en peso de biosólidos incorporados al suelo, para medir el rendimiento del cultivo de rábano (Raphanus sativus), cebolla (Allium cepa) y zanahoria (Daucus carota), resultando la dosis agronómica del $20 \%$ p/p la de mayor rendimiento.
\end{abstract}

Microbiológico, Lodo, Rendimiento

\begin{abstract}
Sludge and biosolids samples were analyzed microbiologically according to NOM-004-SEMARNAT2002, of the Intermunicipal System of Wastewater Treatment and Disposal of the municipalities of Rincon (SITRATA) for a period of 10 months in 2017, the most were found within the LMP according to regulations. In addition, it was detected that the use of peracetic acid in doses greater than $0.1 \% \mathrm{v} / \mathrm{v}$ may be an alternative disinfection in its use in sludge, to reduce the microbiological load, before being used in crops. Physicochemical analyzes were performed on samples of mud and soil to determine their $\mathrm{N}$ and $\mathrm{P}$ content, finding that the solid mud has $1.19 \%$ of $\mathrm{N}$ and $3.51 \%$ of $\mathrm{P}_{2} \mathrm{O}_{5}$ determined on a dry basis; which correspond to high values of these macronutrients, essential for crops. Greenhouse tests were carried out with application doses of 0 to 100 percent by weight of biosolids incorporated into the soil, to measure the crop yield of radish (Raphanus sativus), onion (Allium cepa) and carrot (Daucus carota), resulting in Agronomic dose of $20 \% \mathrm{w} / \mathrm{w}$ the highest yield.
\end{abstract}

Microbiological, Sludge, Yield

Citación: SOTO-ALCOCER, José Luis, RIVERA-MOSQUEDA, Ma. Cruz, GONZÁLEZ-PONCE, María del Refugio y AYALA-ISLAS, Alberto. Análisis Microbiológico de Biosólidos para su aprovechamiento en cultivos de tallo corto. Revista de Ingeniería Biomédica y Biotecnología. 2019. 3-7: 31-38

\footnotetext{
* Correspondencia del Autor (josoto@itesi.edu.mx)

$\dagger$ Investigador contribuyendo como primer autor
} 


\section{Introducción}

El Sistema Intermunicipal para los Servicios de Tratamiento y Disposición de Aguas Residuales para los Municipios del Rincón (SITRATA), es una magna obra de tratamiento, que consiste en el diseño, construcción, equipamiento, puesta en servicio, estabilización y operación transitoria; así como cogeneración de energía eléctrica (SITRATA, 2019).

Su proceso es de lodos activados, con tratamiento anaerobio para cogeneración de energía eléctrica. Al aprovechar el biogás, se contribuye al cuidado del medio ambiente, además de que se tiene un ahorro en gastos de operación de la planta al producir parte de la energía requerida. La calidad del agua tratada cumple con la normatividad, y sus lodos se pueden utilizar en el mejoramiento de los terrenos agrícolas. Esta obra beneficia a 114 mil habitantes de las cabeceras municipales de San Francisco y Purísima del Rincón (Comisión Estatal del Agua, s.f.).

De acuerdo con Oliva (2012), la capacidad de tratamiento de aguas residuales destinada a SITRATA sería de 250 litros por segundo, de los cuales 80 litros corresponden al Municipio de Purísima y 170 litros al Municipio de San Francisco del Rincón. No obstante los beneficios que brinda la planta de tratamiento de aguas a ambos municipios, surge el problema de la disposición de sus lodos, los cuales pueden aprovecharse como abono para plantas de tallo corto.

\section{Justificación}

El agua contaminada proveniente principalmente de las industrias y de las actividades domésticas, se conoce como agua residual, las Plantas de Tratamiento de Aguas Residuales (PTAR) son construidas para el saneamiento de este tipo de agua. Cada día son necesarias más PTAR, y las existentes buscan mejorar el proceso de eliminación de los contaminantes o de tratar un mayor volumen de agua. La acción de la recuperación del agua a su ciclo natural conlleva un impacto ecológico, debido a que los contaminantes retirados del agua residual en estos procesos, pasan a ser parte de los lodos residuales de las PTAR; por lo tanto es necesario tratarlos mediante; métodos de reducción de volumen, técnicas de reducción de patógenos y métodos de estabilización.
Las PTAR están buscando distintos tipos de aprovechamiento de los biosólidos para que el impacto ambiental positivo del agua tratada, no se vea afectado por un nuevo residuo, sino sea parte adicional como sub-producto apto de aprovechamiento (Organización de las Naciones Unidas, 2017). Esto conduce a caracterizar los biosólidos para clasificarlos y conocer el medio por el cual van a poder ser aprovechados. Dentro de la actividad más común de aprovechamiento es su empleo como abono en la agricultura, como cultivos de tallo largo y tallo corto. Para estos propósitos deben de cumplir con características fisicoquímicas (contenido de metales pesados) y microbiológicos (patógenos presentes).

$\mathrm{Si}$ los biosólidos son utilizados inmediatamente para cosecha sin regulación alguna, representarían un riesgo, por ser una vía de contaminación microbiológica y por afectación a los cultivos, por bajos rendimientos de estos.

\section{Hipótesis}

Los biosólidos obtenidos de SITRATA, pueden aprovecharse como abono para cultivos de tallo corto sin afección a los mismos, ni al suelo donde son aplicados.

\section{Objetivo General}

Analizar biosólidos microbiológicamente para su aprovechamiento agrícola en una hortaliza.

\section{Objetivos Específicos}

- Determinar la calidad de los biosólidos de acuerdo a los indicadores de contaminación microbiológica por coliformes fecales, Salmonella spp. y huevos de helminto.

- Implementar una técnica de reducción de patógenos para lodos

- Analizar los nutrientes esenciales de los biosólidos

- Determinar la tasa de aplicación de los biosólidos para los cultivos hortícolas seleccionados mediante una prueba en invernadero. 


\section{Marco Teórico}

El tratamiento de las aguas residuales conduce a la producción de subproductos conocidos como lodos o fangos. Cabe distinguir entre «lodos primarios» es decir los sólidos decantados en el tratamiento primario y «lodos secundarios o biológicos» o bien los sólidos decantados en el clarificador tras el paso de las aguas por el reactor biológico (Secretariado Alianza por el Agua/ Ecología y Desarrollo, 2008). A estos residuos también se les conoce como biosólidos, los que pueden ser benéficamente aprovechados (EPA, 1994a).

La Norma Oficial Mexicana NOM-004SEMARNAT-2002, cita la definición de biosólidos como: «Lodos que han sido sometidos a procesos de estabilización y que, por su contenido de materia orgánica, nutrientes y características adquiridas después de su estabilización, puedan ser susceptibles de aprovechamiento».

Y la misma norma define el aprovechamiento de los biosólidos como: «el uso de los biosólidos como mejoradores o acondicionadores de los suelos por su contenido de materia orgánica y nutrientes, o en cualquier actividad que represente un beneficio».

Particularmente en suelos que son bajos en materia orgánica, los biosólidos proporcionan beneficios que no están disponibles a partir de la fertilización química. El contenido de materia orgánica de biosólidos mejora los medios de enraizamiento del suelo proporcionando una mejor retención de agua, mejor intercambio de aire alrededor de las raíces de las plantas y una mayor capacidad del suelo para mantener los nutrientes en un estado disponible para la planta (EPA, 1994a).

Los beneficios e impedimento del uso de los biosólidos en la agricultura de acuerdo a Andersen (2001), se ven directamente afectados por dos aspectos: el económico, pues sugiere el uso de fertilizantes a bajo costo y el sanitario, pues existe un temor que el uso de lodo en la agricultura podría no ofrecer garantías suficientes con respecto a la seguridad alimentaria. Sin embargo el empleo de ácido peracético puede resultar un desinfectante de alto nivel (Flores et al., 2011).

\section{Metodología}

Para el estudio se obtuvieron muestras de lodo sólido, lodo sólido secado, suelo, lodo filtrado, lodo digerido (con y sin la adición de ácido peracético), así también como muestras de hortalizas (rábano, zanahoria y cebolla) cultivadas en invernadero con biosólidos solamente.

El lodo sólido o biosólido, filtrado y digerido se muestreó en la dirección de camino a San Jerónimo S/N, San Jerónimo, C.P. 36407, Purísima del Rincón, Guanajuato en la compañía del Sistema Intermunicipal para los Servicios de Tratamiento y Disposición de Aguas Residuales para los Municipios del Rincón del sitio de almacenamiento temporal de lodo deshidratado (por debajo de la descarga del filtro prensa) en la

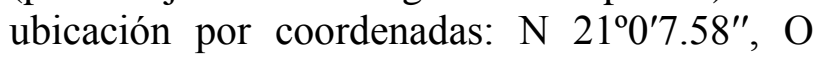
$101^{\circ} 52^{\prime} 38.826^{\prime \prime}$ a $1750 \mathrm{~m}$.

Las muestras de lodo sólido, filtrado y digerido se obtuvieron en condiciones climatológicas donde estaba parcialmente nublado y soleado habiéndose obtenido en distintas horas durante la mañana de los días de análisis.

Debido a la operación discontinua del filtro prensa, la cantidad de lodos en el sitio de almacenamiento temporal de lodo deshidratado es muy variable por lo cual las muestras obtenidas de lodo sólido provienen de montículos de lodo de distinto tamaño.

El lodo sólido secado se obtuvo a partir del secador de bandejas Armfield UOP8-A operado a $40^{\circ} \mathrm{C}$ por 4.5 horas La muestra de suelo se obtuvo de un terreno agrícola en la ubicación por coordenadas: N 20 19'31.655", O $101^{\circ} 36$ '58.534" a $1690 \mathrm{~m}$, de acuerdo a la NOM-021-SEMARNAT-2002 (Fernández, 2002).

El lodo digerido se obtuvo del tanque del reactor anaerobio posterior al digestor anaerobio. Este tipo de lodo se almacena durante periodos indistintos hasta que se acumule la suficiente cantidad para iniciar operación del filtro prensa y ser deshidratados. Al mismo tipo de lodo se le adicionó una dosis de ácido peracético de $0.1 \%$ v/v para su obtención en el laboratorio. 
Las muestras de hortalizas se obtuvieron a partir de pruebas de invernadero con la aplicación de biosólidos al suelo en diferentes dosis. Se toman solamente para el análisis las hortalizas con una posible carga microbiológica mayor; las de los cultivos sembrados solamente con biosólidos.

Para esto se eligieron como cultivos representantes; el rábano variedad Raphanus sativus, cebolla de cambray variedad Allium cepa y zanahoria variedad Daucus carota.

Las muestras así obtenidas se etiquetaron en orden alfabético respecto a las fechas de muestreo o entrada, establecidas desde la primera fecha de muestreo (02 de febrero de 2017) nombrada como muestra A, hasta la última fecha de muestreo (13 de noviembre de 2017), nombrada muestra L, de acuerdo a la Tabla 1.

\begin{tabular}{|l|r|c|}
\hline \multicolumn{1}{|c}{ Identificación de muestra } & $\begin{array}{l}\text { Fecha de } \\
\text { muestreo }\end{array}$ & Muestra \\
\hline Lodo sólido & $02 / 02 / 2017$ & $\mathrm{~A}$ \\
\hline Lodo sólido & $29 / 05 / 2017$ & $\mathrm{~B}$ \\
\hline Lodo sólido & $03 / 07 / 2017$ & $\mathrm{C}$ \\
\hline Lodo sólido secado & $03 / 07 / 2017$ & $\mathrm{D}$ \\
\hline Suelo & $17 / 07 / 2017$ & $\mathrm{E}$ \\
\hline Lodo filtrado & $09 / 10 / 2017$ & $\mathrm{~F}$ \\
\hline Lodo filtrado & $17 / 10 / 2017$ & $\mathrm{G}$ \\
\hline Lodo digerido & $24 / 10 / 2017$ & $\mathrm{H}$ \\
\hline $\begin{array}{l}\text { Lodo digerido+ácido } \\
\text { peracético }\end{array}$ & $24 / 10 / 2017$ & $\mathrm{I}$ \\
\hline Rábano (Raphanus sativus) & $13 / 11 / 2017$ & $\mathrm{~J}$ \\
\hline Zanahoria (Daucus carota) & $13 / 11 / 2017$ & $\mathrm{~K}$ \\
\hline $\begin{array}{l}\text { Cebolla de cambray (Allium } \\
\text { cepa) }\end{array}$ & $13 / 11 / 2017$ & $\mathrm{~L}$ \\
\hline
\end{tabular}

Tabla 1 Identificación de muestras

El método de muestreo, conservación y trasporte y análisis de las muestras de lodos y biosólidos se realizó de acuerdo a la NOM-004SEMARNAT-2002 (SEMARNAT, 2002).

A las muestras obtenidas, se les determinó el contenido de sólidos totales (ST) en porcentaje en peso, en un tiempo máximo de análisis de 24 horas, mediante el uso de una termobalanza modelo A\&D MF-50.

A las muestras de lodo y biosólidos se les determinó la concentración de patógenos y parásitos (coliformes fecales, Salmonella spp. y huevos de helminto) como se indica en la normatividad: NOM-004-SEMARNAT-2002 (SEMARNAT, 2002).
En los análisis fisicoquímicos se determinó el contenido de nitrógeno total y fósforo total $\left(\mathrm{P}_{2} \mathrm{O}_{5}\right)$ a las muestras $\mathrm{C}, \mathrm{D}$ y $\mathrm{E}$ mediante el «Fotómetro multiparámetrico Hanna modelo HI83214 C214».

Los biosólidos de la muestra B y el suelo, muestra $\mathrm{C}$, se utilizaron para las pruebas en invernadero. Se realizaron 11 tratamientos de 0 a 100 en porcentaje en peso húmedo de biosólidos aplicados al suelo. Se mezcló perfectamente los biosólidos con el suelo representativo de la región y se eligió como cultivos representantes al rábano (Raphanus sativus), cebolla de cambray (Allium cepa) y zanahoria (Daucus carota) registrándose el peso, largo y ancho de las hortalizas para determinar el rendimiento del cultivo.

\section{Resultados}

En la Tabla 2 se puede observar la comparación de las muestras con los Límites Máximos Permisibles (L.M.P) de acuerdo a normatividad. La muestra B analizada, que corresponde a lodo sólido, se encuentra en los límites respecto a las tres determinaciones.

La muestra $\mathrm{C}$ analizada, correspondiente a lodo sólido obtenido en diferente fecha de muestreo del mismo sitio, se encuentra por encima de los límites máximos permisibles respecto a coliformes fecales y salmonella spp.

Sin embargo, la muestra D que es un lodo sólido que ha sido sometido a un proceso de secado, se encuentra por debajo de los L.M.P. en las tres determinaciones de acuerdo a normatividad.

Para un estudio más completo se procedió al análisis de lodos de diferentes sitios en la PTAR. Para esto se obtuvieron 2 muestras de lodo filtrado (muestras F y G), una muestra de lodo digerido (muestra $\mathrm{H}$ ) y una muestra preparada a partir de la muestra $\mathrm{H}$, pero con la adición de ácido peracético en dosis $0.1 \%$ v/v, mostrados en la Tabla 3.

Las muestras $\mathrm{F}$ y $\mathrm{G}$ obtenidas de la descarga directa del filtro prensa, son representativas de los biosólidos generados en la compañía de SITRATA, se encuentran dentro de la normatividad, en la clase $\mathrm{C}$ y debajo de los L.M.P. de acuerdo a las tres determinaciones de indicadores de patógenos y parásitos. 
La Tabla 4 presenta los valores de las determinaciones microbiológicas realizadas para su comparación a la literatura de las muestras de rábano, zanahoria y cebolla; muestras $\mathrm{J}, \mathrm{K}$ y L respectivamente, obtenidas de las pruebas en invernadero con biosólidos solamente.

Moragas \& Pablo, (2017) establecen una recopilación de normas microbiológicas para alimentos, en este caso consideran los L.M.P. establecidos por Rosario Pascual en su libro «Microbiología Alimentaria», los cuáles se denotan para verduras y hortalizas.

\begin{tabular}{|c|c|c|c|c|c|c|c|}
\hline \multirow[t]{2}{*}{$\begin{array}{l}\text { Determinac } \\
\text { ión }\end{array}$} & \multicolumn{4}{|c|}{$\begin{array}{c}\text { Resultados } \\
\text { Base seca }\end{array}$} & \multicolumn{3}{|c|}{$\begin{array}{l}\text { L.M.P. NOM-004- } \\
\text { SEMARNAT-2002 (base } \\
\text { seca) }\end{array}$} \\
\hline & $\begin{array}{c}\text { Muest } \\
\text { ra A }\end{array}$ & $\begin{array}{c}\text { Muest } \\
\text { ra B }\end{array}$ & $\begin{array}{c}\text { Muest } \\
\text { ra C }\end{array}$ & $\begin{array}{c}\text { Muest } \\
\text { ra D } \\
5.50\end{array}$ & $\begin{array}{c}\text { CLAS } \\
\text { E A }\end{array}$ & $\begin{array}{c}\text { CLAS } \\
\text { E B }\end{array}$ & $\begin{array}{c}\text { CLAS } \\
\text { E C }\end{array}$ \\
\hline $\begin{array}{r}\% \text { Humedad } \\
1 . \mathrm{NMP} / \mathrm{g} \mathrm{ST}\end{array}$ & $\begin{array}{l}\frac{80.73}{\geq 2} \\
\geq 2\end{array}$ & $\frac{80.95}{<}$ & $\begin{array}{l}82.36 \\
\geq 2\end{array}$ & $\begin{array}{l}5.99 \\
240\end{array}$ & $\begin{array}{c}\mathrm{EA} \\
<1\end{array}$ & $\begin{array}{r}\mathrm{EB} \\
<1\end{array}$ & $\begin{array}{c}\mathrm{EC} \\
<2\end{array}$ \\
\hline $\begin{array}{l}\text { de Coliformes } \\
\text { Fecales. } \\
\text { NOM-004- } \\
\text { SEMARNAT } \\
-2002\end{array}$ & $\begin{array}{l}400 \\
000\end{array}$ & 3000 & $\begin{array}{l}400 \\
000\end{array}$ & 000 & 000 & 000 & $\begin{array}{l}000 \\
000\end{array}$ \\
\hline $\begin{array}{l}\text { 2. NMP/g ST } \\
\text { de Salmonella } \\
\text { spp. NOM- } \\
\text { 004- } \\
\text { SEMARNAT } \\
\text {-2002 }\end{array}$ & - & $<300$ & 1500 & $<300$ & $<3$ & $<3$ & $<300$ \\
\hline $\begin{array}{l}\text { 3. Huevos de } \\
\text { Helminto en } \\
\text { 2g de ST. } \\
\text { NOM-004- } \\
\text { SEMARNAT } \\
-2002\end{array}$ & - & $<1$ & 6 & 1 & $\begin{array}{l}<1 \\
\text { (a) }\end{array}$ & $<10$ & $<35$ \\
\hline
\end{tabular}

Tabla 2 Análisis microbiológico de biosólidos de acuerdo a la NOM-004-SEMARNAT-2002

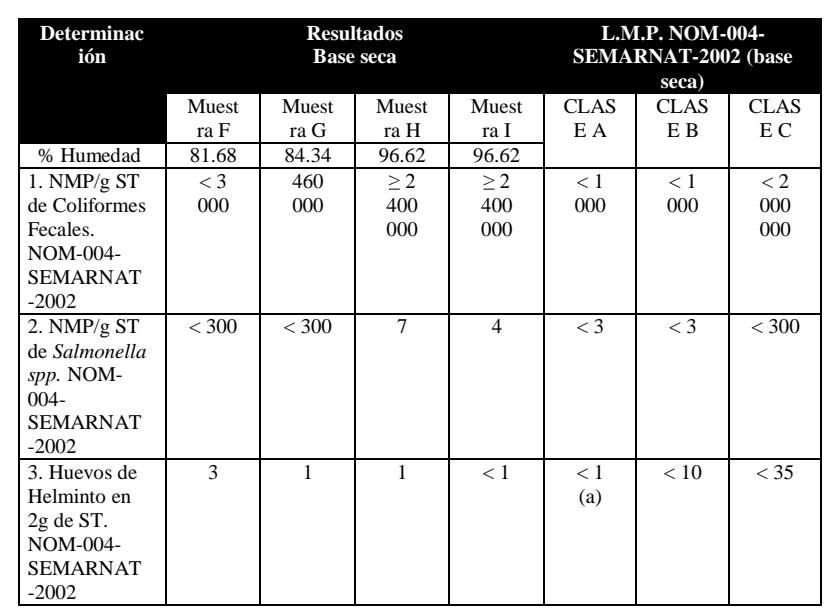

Tabla 3 Análisis microbiológico de lodos de acuerdo a la NOM-004-SEMARNAT-2002

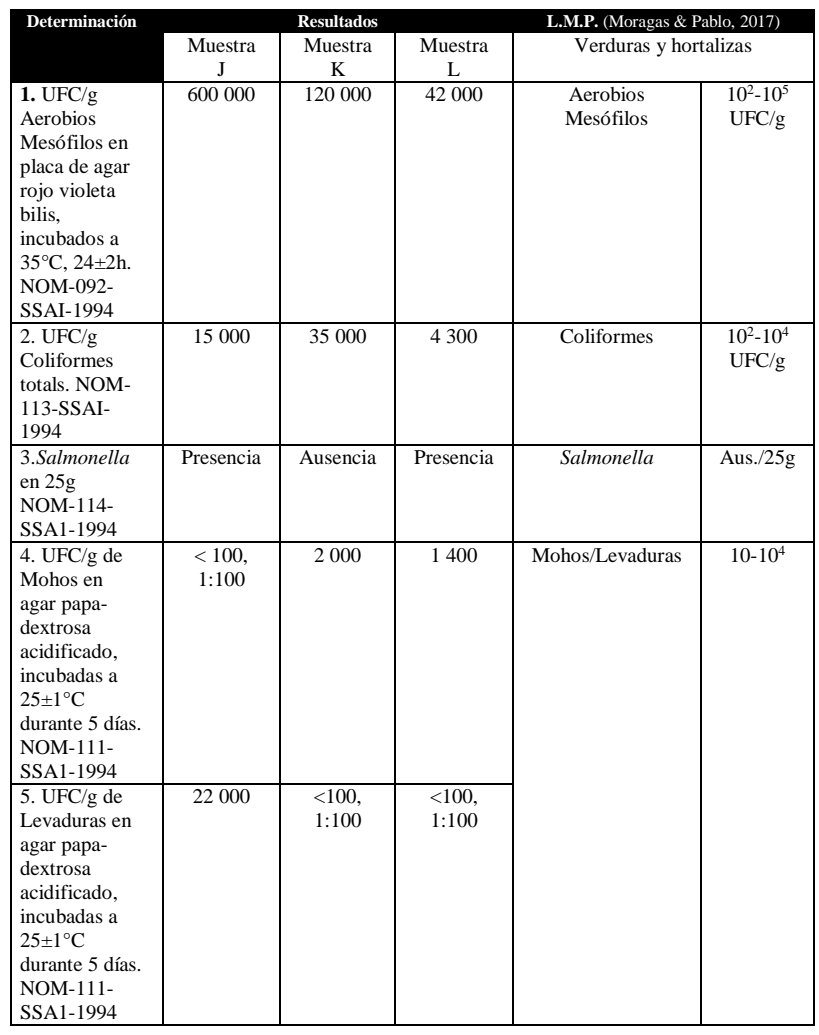

Tabla 4 Análisis microbiológico de rábano, zanahoria y cebolla

Por lo anterior comparando los datos obtenidos de las determinaciones realizadas a las muestras $\mathrm{J}, \mathrm{K}$ y L, se encuentra que las tres muestras no se encuentran por debajo de los L.M.P. de al menos una de las determinaciones realizadas, de acuerdo a la literatura citada.

La muestra $\mathrm{J}$ se presenta con una cantidad elevada de mesófilas aerobias, presencia de Salmonella en 25 gramos y de Levaduras. La muestra de zanahoria (muestra K) exhibe una alta cantidad de coliformes totales, así también como la muestra de cebolla (muestra L) indica presencia de salmonella.

Las muestras analizadas son obtenidas a partir de pruebas de invernadero utilizando biosólidos solamente como medio de crecimiento, este método representa el caso de que los biosólidos fuesen utilizados de manera inmediata, sin suelo y sin algún tipo de regulación. En definitiva, representan un riesgo por una vía de contaminación microbiológica sólo en caso de utilizarse de manera inmediata.

Las técnicas de aplicación y uso seguro de biosólidos de acuerdo a la EPA (2004b), promueven una mejor calidad de los cultivos que son cosechados en suelos a los que se les ha aplicado biosólidos. 
Para el caso de la utilización de biosólidos o lodos de una PTAR en la agricultura, resulta interesante conocer el contenido de $\mathrm{N}$ y $\mathrm{P}$ en ellos, pues resultan ser unos de los elementos más importantes para las plantas. Para esto se procedió a un análisis fisicoquímico de biosólidos (muestra C), biosólidos secos (muestra D) y suelo (muestra E).

En la Tabla 5 se muestran los datos del contenido de nitrógeno total y fósforo total en forma de ácido fosfórico $\left(\mathrm{P}_{2} \mathrm{O}_{5}\right)$. La muestra $\mathrm{C}$ cuenta con un contenido de nitrógeno y con un alto contenido de fósforo, respecto a la literatura citada. Por el contenido de sólidos totales el tipo de lodo se puede denominar de tipo torta.

La muestra D aún cuenta con contenido de nitrógeno y fósforo más altos que la muestra de suelo (muestra E), y la determinación del contenido de nitrógeno y fósforo a los biosólidos secos, muestra que para la aplicación de algún tipo de secado pueden existir pérdidas por volatilización tanto de nitrógeno como fósforo.

Se puede destacar que el contenido de nitrógeno y fósforo en lodos no es comparable con el de los fertilizantes químicos, sin embargo, los lodos presentan una larga disponibilidad de estos en el suelo.

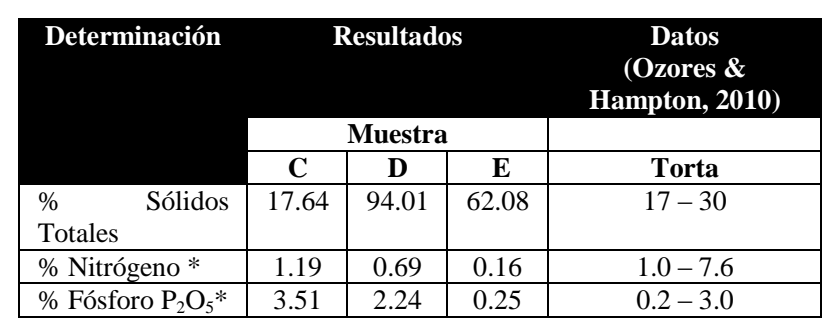

*Determinado en base seca, kilogramos del elemento $\mathrm{N}$ por $100 \mathrm{~kg}$ de muestra o kilogramos de P2O5 por $100 \mathrm{~kg}$ de muestra

Tabla 5 Análisis de nitrógeno total y fósforo total en biosólidos y suelo

Los estudios correspondientes a las pruebas de invernadero arrojaron los rendimientos obtenidos (reflejados principalmente en el peso), al combinar los biosólidos con el suelo de la región; respecto al rábano, se obtuvieron mayores rendimientos, en comparación con el control (0 \% biosólidos, 100 $\%$ suelo) en las dosis de aplicación de $20 \%$ y 70 $\%$, como se aprecia en la Figura 6.
En cuanto al rendimiento de la zanahoria, mostrado en la Figura 7, se puede notar que hubo un mayor rendimiento en las mezclas de 20, 30, 40 y $60 \%$ p/p con respecto al control. Los mayores rendimientos de los cultivos se observan en las dosis de aplicación menor a 60 $\%$. Como se puede observar en la Figura 8 , hubo un aumento en el rendimiento de la cebolla de cambray en las mezclas con 20, 90 y $100 \%$ p/p de biosólidos incorporados al suelo.

Cabe destacar que la disminución del rendimiento en las mezclas con mayor porcentaje de biosólidos, ocurre a partir de $60 \%$ $\mathrm{p} / \mathrm{p}$ principalmente, en los tres casos.

\section{Conclusiones}

De acuerdo a los análisis microbiológicos realizados al lodo sólido, seco y filtrado (con y sin adición de ácido peracético, conforme a la NOM-004-SEMARNAT-2002, de SITRATA de la ciudad de Purísima del Rincón Guanajuato, en un lapso de 10 meses del año 2017, se encontraron algunas variaciones en los resultados obtenidos, no obstante la mayoría de los análisis estaban dentro de la normatividad.

El uso de ácido peracético en dosis mayores que $0.1 \% \mathrm{v} / \mathrm{v}$ puede resultar como una alternativa de desinfección en su uso en lodos, para disminuir la carga microbiológica, antes de ser utilizados en cultivos.

Los análisis fisicoquímicos a muestras de lodo y suelo para determinar su contenido de $\mathrm{N}$ y P demostró que la muestra C contó con $1.19 \%$ de $\mathrm{N}$ y $3.51 \%$ de $\mathrm{P}_{2} \mathrm{O}_{5}$ determinado en base seca; que corresponden a valores altos de estos macronutrientes, indispensables para los cultivos.

La prueba en invernadero con dosis de aplicación de 0 a 100 en porcentaje en peso de biosólidos incorporados al suelo, ha demostrado que existe variación respecto al rendimiento del cultivo de rábano (Raphanus sativus), cebolla (Allium cepa) y zanahoria (Daucus carota). Sin embargo, la dosis agronómica de mayor rendimiento de los tres cultivos resultó ser de 20 $\% \mathrm{p} / \mathrm{p}$. 
A los cultivos cosechados solamente con biosólidos se realizó análisis microbiológico para demostrar posibles vías de contaminación microbiológica por el uso del biosólidos de la planta de tratamiento de aguas residuales en su aprovechamiento en usos agrícolas. Los cultivos cosechados no cumplieron con la normatividad respecto a los L.M.P de acuerdo a la literatura citada.

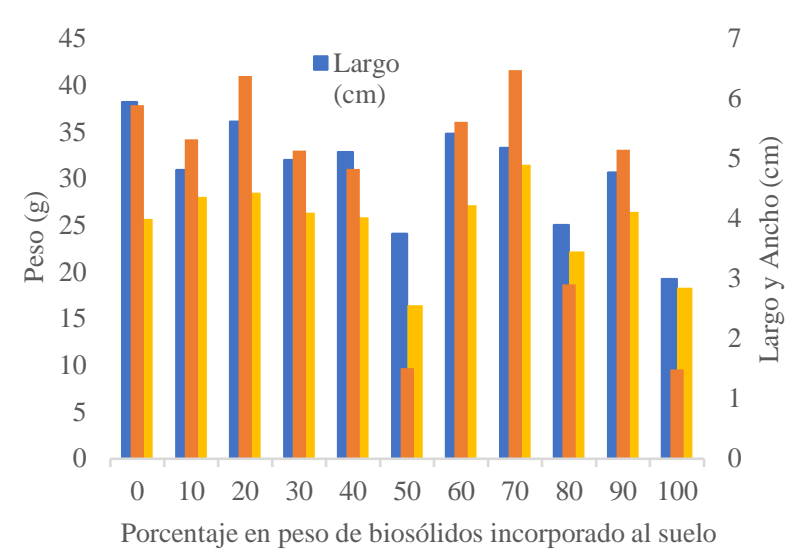

Figura 6 Rendimiento de rábano (Raphanus sativus) con dosis creciente de biosólidos aplicado al suelo

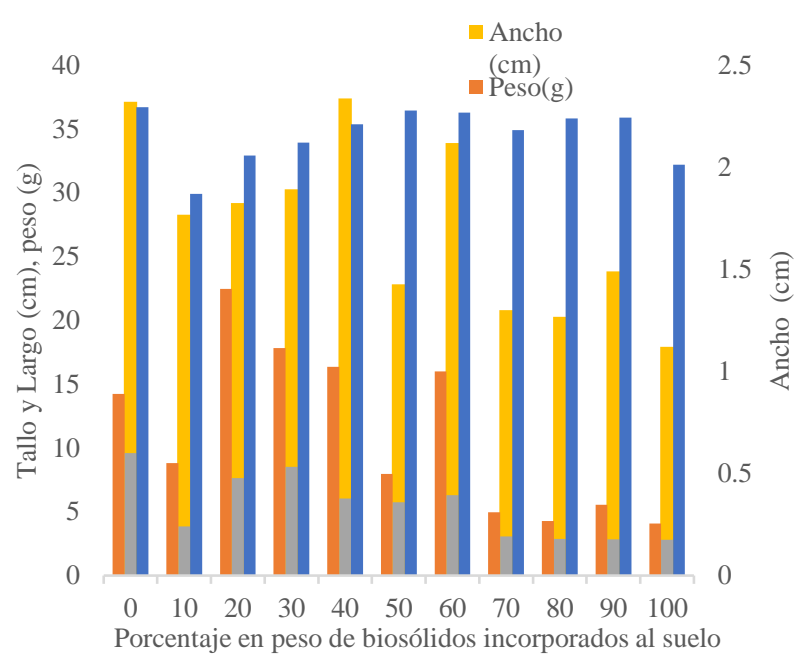

Figura 7 Rendimiento de zanahoria (Daucus carota) con dosis creciente de biosólidos aplicado al suelo

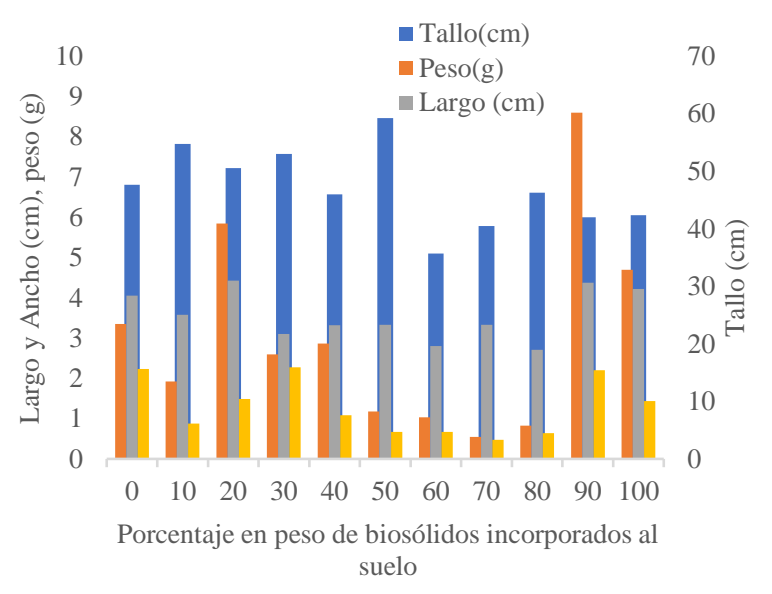

Figura 8 Rendimiento de cebolla de cambray (Allium сера) con dosis creciente de biosólidos aplicados al suelo

\section{Agradecimientos}

El presente estudio se realizó con la colaboración del personal del Sistema Intermunicipal para los Servicios de Tratamiento de Aguas Residuales para los Municipios del Rincón (SITRATA), del municipio de Purísima del Rincón, Guanajuato (Convenio ITESI/CONVENIOS/240-2017). El Instituto Tecnológico Superior de Irapuato agradece a todos ellos y a su Director General: Ing. Diego Isaac Dávila Cano, todo el apoyo otorgado para la realización de este trabajo.

\section{Referencias}

Andersen, A. (Octubre de 2001). Disposal and recycling routes for sewage sludge. Obtenido de europa:

http://europa.eu.int/environment/pubs/home.ht $\mathrm{m}$.

Comisión Estatal del Agua. (s.f.). Gobierno del Estado de Guanajuato. Recuperado el 10 de Noviembre de 2017, de Comunidad sustentable: http://www.guanajuato.gob.mx/ceag/plantas.ph p.

EPA. (1994a). Biosolids recycling: Beneficial technology for a better enviroment. Washington DC: Office of water.

EPA. (1994b). A plain english guide to the EPA part 503 biosolids rule. Washington, DC: U.S. Enviromental Protección Agency Office of Wastewater Management.

Fernández, C. L. (31 de Diciembre de 2002). Norma Oficial Mexicana NOM-021SEMARNAT-2000 que establece las especificaciones de fertilidad, salinidad y clasificación de suelos, estudio, muestreo y análisis. Distrito Federal, Estado de México, México.

Flores, M., Nieres, P., E., A., Cassano, \& Labas, M. D. (2011). Desinfección de agua con ácido peracético: una alternativa económica y ecológica. Santa Fe, Argentina, América del Sur.

Moragas, M. E., \& Pablo, B. B. (1 de Enero de 2017). Recopilación Normas Microbiológicas de los alimentos y asimilados (superficies, aguas diferentes de consumo, aire, subproductos) otros parámetros físico-químicos de interés sanitario. Bilbao, España, Europa.

SOTO-ALCOCER, José Luis, RIVERA-MOSQUEDA, Ma. Cruz, GONZÁLEZ-PONCE, María del Refugio y AYALA-ISLAS, Alberto. Análisis Microbiológico de Biosólidos para su aprovechamiento en cultivos de tallo corto. Revista de Ingeniería Biomédica y Biotecnología. 2019 
Oliva, M. L. (14 de diciembre de 2012). Inicia operaciones planta tratadora. El sol de León.

Organización de las Naciones Unidas. (2017).

AGUAS RESIDUALES EL RECURSO NO

EXPLOTADO [Informe]. Recuperado 22 agosto, 2019, de http://aneas.com.mx/wpcontent/uploads/2017/03/247647S.pdf.

Secretariado Alianza por el Agua/ Ecología y Desarrollo. (2008). Manual de Depuración de Aguas Residuales Urbanas. España: Ideasamares.

SEMARNAT. (24 de Septiembre de 2002). NORMA Oficial Mexicana NOM-004SEMARNAT-2002, Protección Ambiental.Lodos y Biosólidos.- Especificaciones y límites máximos permisibles de contaminantes para su aprovechamiento y disposición final. Distrito Federal, México. 